\title{
Regulation of Extracellular Matrix in C. elegans: Lessons in Sweating the Small Stuff
}

\author{
R.D. Schultz ${ }^{1,2}$, E.A. Ellis ${ }^{3}$, and T.L. Gumienny ${ }^{4}$ \\ 1. Molecular and Cellular Medicine, Texas A\&M University Health Science Center, College Station, TX, \\ USA. \\ 2. The Brown Foundation Institute of Molecular Medicine, University of Texas Health Science Center, \\ Houston, TX, USA. \\ ${ }^{3 .}$ Microscopy and Imaging Center, Texas A\&M University, College Station, TX, USA. \\ 4. Department of Biology, Texas Woman's University, Denton, TX, USA.
}

In humans and other mammals, environmental sensing, extracellular matrix, and signaling pathways can affect body size. Many of these modes of body size regulation are conserved in the small free-living nematode $C$. elegans. One of the critical factors affecting body size is the bone morphogenetic protein (BMP) pathway, which is a family of signaling molecules that is widely conserved [1]. In mammals, BMP pathway signaling is important for the growth and homeostasis of extracellular matrix, including basement membrane remodeling, and long bone growth. In C. elegans, BMP signaling regulates body size development and drug response via unknown physiological mechanisms. Our lab and others have shown animals with decreased BMP signaling ( $b m p(-))$ have a short body length, while animals overproducing BMP $((b m p(++))$ are long [2]. Large-scale gene expression studies have shown that BMP pathway signalling regulates a multitude of genes involved in body size determination [3-4]. Further, the nematode cuticle, which is an extracellular matrix that surrounds and protects the animal from its environment, can influence body shape [5]. Using both light and electron microscopy methods, we show that BMP regulates body length through organization and composition of the nematode cuticle, which may provide an explanation for both body size and drug response traits associated with this signaling pathway.

The nematode cuticle is patterned by circumferential rings called annuli. Using a vital lipophilic dye, we stained the cuticle of animals with varied BMP dose and found that BMP affects cuticle shape. Animals with decreased BMP signaling $(b m p(-))$ have a decreased annular width, while long animals with increased BMP signaling $(b m p(++))$ have an increased annular width, proportional to their total body length (Fig. 1). These results indicate that BMP signaling affects the shape of the cuticle, where small $b m p(-)$ animals possess a condensed cuticle and $b m p(++)$ animals exhibit an expanded cuticle.

We also examined the cuticle organization and composition using TEM. Because the cuticle is an extremely tough and complex barrier consisting of multiple layers that differ in both composition and organization, we optimized sample preparation in a few ways. First, we used a microwave processor, increasing the rate of molecular diffusion along the entire animal and thus eliminating any need for cuticle cutting during specimen preparation. Next, we stained the animals with malachite green, which acts to further differentiate the cuticular layers and preserve lipids that are normally stripped during dehydration [6]. Finally, we customized the resin formulation to maintain a lower viscosity while matching the significant hardness of the specimens. We found that malachite green stains and binds to lipids on the outermost layer of the cuticle. We observed the deposition of these lipids to be dependent on the dose of BMP, where animals lacking BMP signaling displayed a severely depleted lipid layer and animals with overexpressed BMP signaling had an increased lipid layer as compared to wild type (Fig. 
2). We also found $\operatorname{bmp}(-)$ animals displayed a strikingly dense medial layer compared to wild-type or $b m p(++)$ animals, indicating BMP signaling level affects the composition of the medial layer (Fig. 2). Finally, we discovered that BMP signaling can alter the organization of the basal layer, as the top-most basal layer of oriented collagens is less structured in some $b m p(++)$ animals (Fig. 2). These results reveal a role for BMP signaling in both the organization and composition of the cuticle. We propose that alteration of the cuticle through BMP pathway signaling underlies both body length and drug response phenotypes. Furthermore, we show a conserved function for the BMP family in regulation of extracellular matrix [7].

Note: Ann helped in the design and acquisition of data for this paper prior to her passing on October 29, 2016. Ann was a wonderfully special person and a great mentor. She will be sorely missed.

References:

[1] IL Blitz and KW Cho, Dev Dyn 238 (2009), p. 1321.

[2] RD Schultz et al, PLoS One 9 (2014), e101929.

[3] J Liang et al, Dev Biol 305 (2007), p. 714.

[4] AF Roberts et al, BMC Developmental Biology 10 (2010), p. 61.

[5] L McMahon et al, Mol Biol Cell 14 (2003), p. 1366.

[6] RJ Teichman et al, Biology of Reproduction 7 (1972), p. 73.

[7] This work was supported by NIH 1R01GM097591-01 and by TAMHSC MCMD start-up funds.

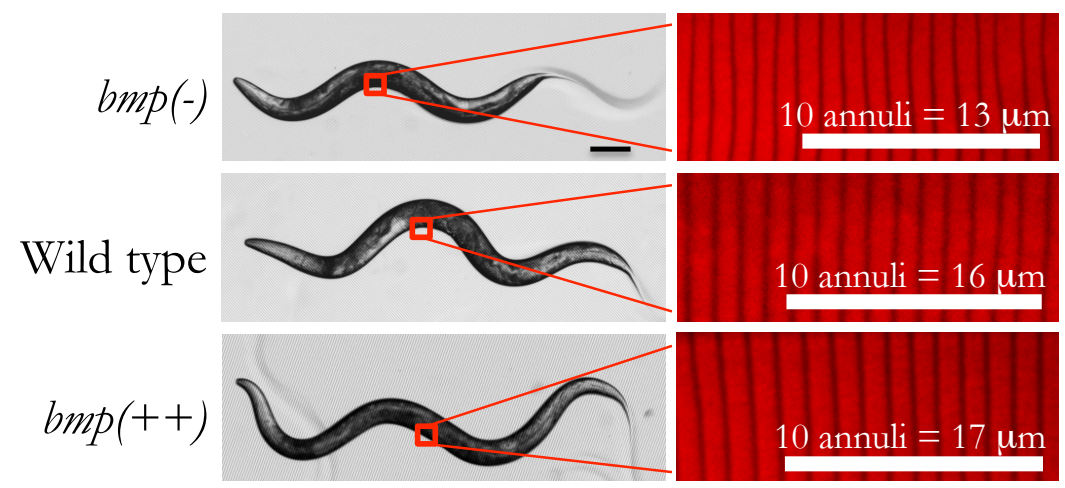

Figure 1. BMP signaling affects body size development, where animals with decreased BMP signaling are small and animals with increased BMP levels are long (left). Scale bar $=100 \mu \mathrm{m}$ (left). Lipid staining of the cuticle in animals with varying BMP levels reveals differences in cuticular shape (right).

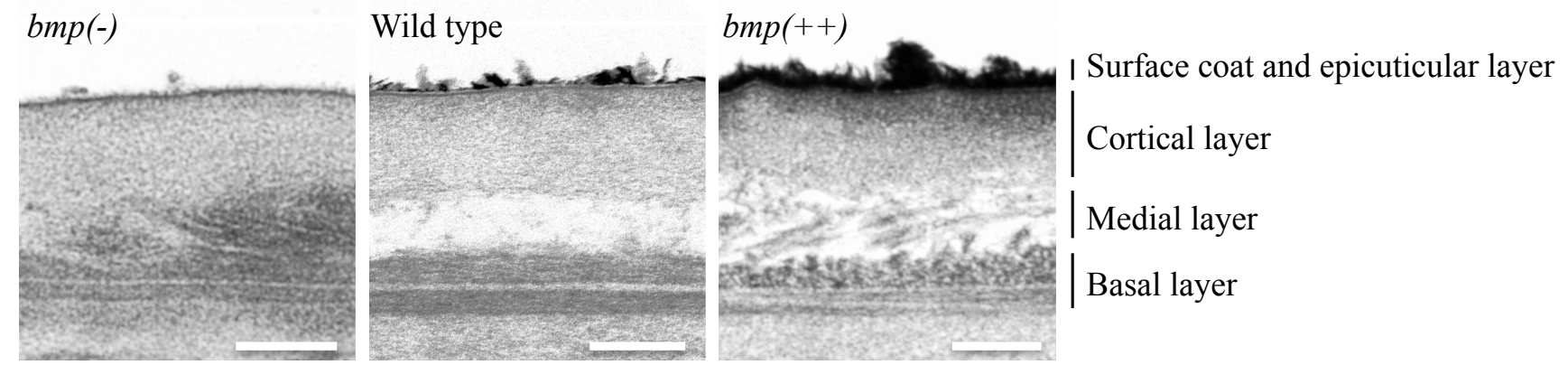

Figure 2. Layer of the cuticle in animals with decreased (left), wild-type (middle), or increased (right) levels of BMP signaling. Scale bars $=0.2 \mu \mathrm{m}$. 